\title{
MACHINERY PRODUCTION LOGISTICS SYSTEM UNDER DATA MINING
}

\author{
Gang Huang 1 \\ ${ }^{1}$ Chongqing Transportation Vocational College, Jiangjin, Chongqing, 402247, China. \\ Email: chongq_huanggang@126.com
}

\begin{abstract}
The research is to improve the working efficiency of transportation vehicles in the mechanical logistics system and maximize the utilization efficiency of resources in various fields, thereby promoting the rapid development of China's logistics enterprises. First, this paper describes the vehicle routing problem solving algorithm with time window in the machinery production logistics system. Secondly, an optimized genetic algorithm is proposed to perform the improvement of the chromosome coding method, the adjustment of the convergence rate, the survival of the fittest of the genetic gene, and the cross-exchange and change of genes. Finally, the optimized genetic algorithm is simulated. Also, the general genetic algorithm and the optimized genetic algorithm are used to calculate the actual transportation route. The research results show that by using the optimized genetic algorithm for the route planning of logistics transportation vehicles, the average distance cost calculated is $143.3 \mathrm{~km}$. The best route is sending 4 transportation vehicles for mechanical transportation and the total distance cost is $119 \mathrm{~km}$. The average transportation cost of the 4 transportation routes is reduced, and the transportation efficiency is high, which can meet the customer's time window limit. Due to the shortcomings of the methods of gene cross-exchange and change, the general genetic algorithm will lead to the loss of good genes, and the calculation efficiency is lower. The optimized genetic algorithm can effectively solve the problem of good gene loss, and the calculation efficiency is significantly improved. The best vehicle transportation route is planned to maximize cost savings while meeting customer requirements. The genetic algorithm in data mining is optimized to smoothly calculate the transportation vehicle route for mechanical production logistics distribution at the lowest cost. Also, it can enable vehicles to complete distribution tasks efficiently, providing a feasible basis for the development of machinery production logistics system.
\end{abstract}

Keywords: Data Mining; Optimized Genetic Algorithm; Machinery Logistics; Transportation Vehicle Route.

\section{Introduction}

With the rapid development of economy, China has made strategic changes in the economic development model and economic structure. Since the beginning of the 21st century, China has invested a lot of development funds in large-scale engineering projects such as basic equipment, highways, mooring ports, and bridges in transportation [1]. The development of construction machinery enterprises taking this opportunity is also booming. Facing the increasingly fierce competition in the construction machinery industry, how to improve the operating efficiency of the machinery production process has become a problem that is widely concerned and studied by various construction machinery enterprises.

Most of the machinery production of construction machinery enterprises are large-scale and heavy machinery, and the transportation process requires a lot of manpower and financial resources. Therefore, the optimization of the operating equipment in the machinery production logistics system to improve production efficiency is the core issue. In the process of machinery production, many materials need to be conveyed and circulated. However, general machinery production lines are cumbersome and flow randomly. Therefore, the use of mathematical analysis methods cannot perform a complete analysis of the entire machinery production process and an optimization research for the logistics adopting an appropriate method in the production line [2]. Data mining technology [3] can be based on a variety of algorithms and networks to cluster analysis of a large number of data in the machinery production logistics system. It provides an effective way to solve problems in the machinery production logistics system.

This paper first describes the vehicle routing problem solving algorithm with time windows in the machinery production logistics system, and proposes 
an optimized genetic algorithm in data mining technology. Based on related theories such as production logistics and data mining, this paper analyzes the characteristics of logistics vehicle scheduling in the machinery production logistics system. Then, the problem of vehicle routing with time windows in the logistics system of machinery production is solved, with improving the working efficiency of transportation vehicles and reducing transportation costs.

\section{Literature Review}

In the research process of production logistics system, the core part is the route planning of logistics transportation vehicles. The application of data mining in the route planning of logistics transportation vehicles has also been analyzed by many researchers.

\subsection{The research status of route planning for logistics transportation vehicles}

Cheng et al. (2015) proposed the optimal allocation method for the purpose of minimizing the cost of logistics transportation in view of the dynamics and uncertainty in the logistics transportation process, the replaceability and constraints of logistics transportation resources. The working hours of transportation vehicles were optimized, and the improved genetic algorithm was able to calculate the best path for logistics transportation routes [4]. Lv and Liu (2016) used Petri net to model and analyze flower production processes in view of some problems in flower production logistics. At the same time, the Witness simulation software is used to study the flower production process, thereby improving the operating efficiency of flower production logistics [5]. Zhang et al. (2018) proposed a data-driven model based on analytic target cascade to solve the problems of long waiting time and serious energy waste in the logistics transportation process. This method can shorten the logistics transportation time and improve the operating efficiency of the logistics system [6].

\subsection{The research status of logistics vehicle route planning with time windows}

With the booming development of online shopping, it is an important issue for logistics enterprises to improve distribution efficiency, reduce logistics costs, and meet actual logistics constraints including vehicle capacity and customer available time windows. That is the vehicle routing problem with time windows (VRPTW). Chen et al. (2016) proposed a time window for vehicle route planning based on a dual-objective evolutionary algorithm in view of the uncertainty of logistics transportation route time. It improved the robustness of logistics transportation route calculation [7]. Yang et al. (2017) proposed an optimized recursive constructor (PRC) method for most algorithms that could not effectively solve the VRPTW problem when the number of goods increased rapidly. Taking into account the complex environment of actual logistics, the logistics route for the rapid increase in the number of goods can be effectively calculated to improve the transportation efficiency of large-scale logistics [8]. With the increase of customer-oriented service strategies to meet more complex and strict customer requirements, meeting predetermined time windows has become an important part of vehicle routing planning for logistics activities. $\mathrm{Hu}$ et al. (2018) proposed a two-stage algorithm based on an improved adaptive variable neighborhood search heuristic to solve the demand from customers. The results show that the algorithm can meet the needs of customers while minimizing transportation costs [9].

\subsection{Summary}

To sum up, there are many researches on data mining in the logistics system, but few researches have been applied to the machinery production logistics system. Therefore, this paper proposes an optimized genetic algorithm for the route planning of transportation vehicles with time windows in machinery production logistics system. Then, it provides a new calculation method for the research of machinery production logistics system.

\section{Methodology}

\subsection{The calculation method of machinery production logistics route with time windows}

In the actual machinery production logistics distribution, customer requirements are not only the number of transportation vehicles, but also the need for time windows. Therefore, the time window is included in the distribution phase of the logistics system. The general customer will require the goods to be delivered within the time period they can receive, which also determines whether the customer is satisfied with this logistics solution. It requires machinery production enterprises to be able to find an appropriate method to balance customer satisfaction and logistics transportation distance [10]. The initial vehicle routing problem with time windows is that the logistics vehicle must deliver the goods at the time requested by the customer. Delayed or early delivery of the goods will make the transaction fail. It is assumed that the given arrival time of the logistics transportation vehicle is 
[m, n]. Delaying or advancing arrival time will generate a corresponding penalty amount $\mathrm{T}$. It is shown in Figure 1.

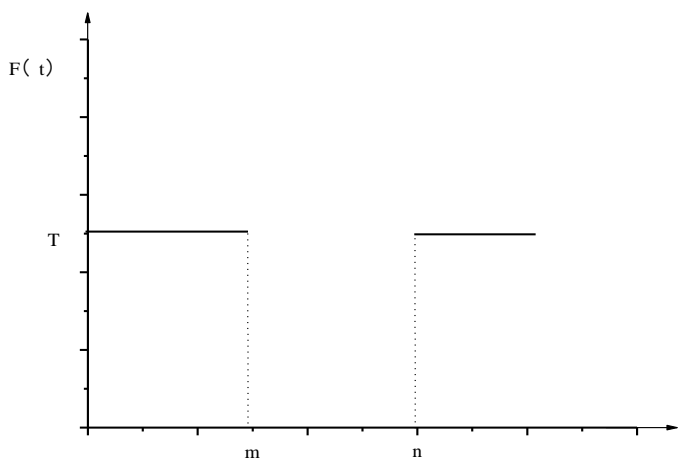

Figure 1: The penalty diagram of time window amount under rigid conditions

However, in actual situations, the complexity of transportation roads is different and the external factors of the force majeure may be encountered during transportation. Therefore, the time window requirements are reduced. When the logistics transportation does not meet the requirements of time windows, the corresponding penalty will be given according to the time of delivery of the goods. The principle of penalty is shown in Figure 2.

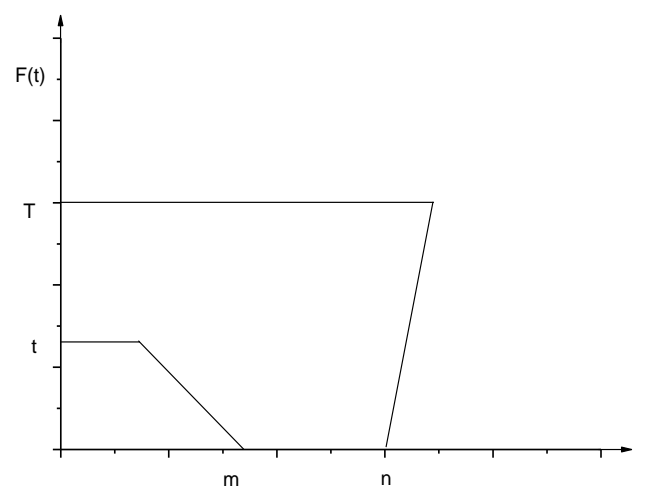

Figure 2: The penalty diagram of time window amount under soft conditions

This research comprehensively analyzes the logistics route in the actual situation, choosing time windows under soft conditions as an additional condition in the logistics transportation process.

\subsection{The optimized genetic algorithm}

The genetic algorithm [11], its core idea is to simulate the evolutionary methods and processes of biological jungle justice. The algorithm can crossexchange, replicate and mutate the superior genes when solving optimization problems. Then, the superior genes can be stably inherited, and the best answer can be found in the continuous evolution process of the organism. When solving the best route selection problem of logistics vehicles, the improved optimization and stability of the genetic algorithm can be used to find the best route selection scheme.

First, a logistics vehicle routing problem model with time windows is established. It is assumed that the logistics distribution has only one centralized warehouse, with $\mathrm{m}$ customers, i identical transportation vehicles, and the transportation vehicle weight $\mathrm{W}$. The time window requested by the customer is $\left[t_{a}, t_{b}\right]$. The transportation vehicles depart from the centralized warehouse and are delivered to the delivery location provided by each customer. The same vehicle can transport machinery to different customers, but the machineries of the same customer cannot be transported separately.

The delivery location of each vehicle cannot be the same. This machinery transportation must be completed on the basis of the shortest transportation route and the least number of logistics vehicles.

The model of logistics vehicle routing problem with time windows is established as follows.

$$
\begin{aligned}
& E=\min \left(E_{1}+E_{2}\right) \\
& E_{1}=\sum_{y=1}^{\mathrm{r}} \sum_{u=1}^{r} \sum_{o=1}^{i} p_{y u} \times a_{y u} \times s_{y u o} \\
& E_{2}=\sum_{0-1}^{i} F\left(g_{y}\right) \\
& \sum_{y=1}^{r} h_{y} j_{y o} \leq K, o=1,2 \cdots, i \\
& \sum_{o=1}^{i} j_{y o}=1, o=1,2 \cdots, \mathrm{r} \\
& \sum_{y=0}^{r} s_{y u o}=j_{u o}, \mathrm{u}=1,2 \cdots, \mathrm{r}, \mathrm{o}=1,2 \cdots, \mathrm{i} \\
& \sum_{u=0}^{r} s_{y u o}=j_{y o}, \mathrm{u}=1,2 \cdots, \mathrm{r}, \mathrm{o}=1,2 \cdots, \mathrm{i} \\
& v t_{y}^{<t_{y}<c t} t_{y} \\
& \sum_{u=0}^{r} s_{0 u o}=1, \mathrm{u}=1,2 \cdots, \mathrm{r}, \mathrm{o}=1,2 \cdots, \mathrm{i} \\
& \sum_{y=0}^{r} s_{y 0 o}=1, \mathrm{u}=1,2 \cdots, \mathrm{i}
\end{aligned}
$$

Where: Equations (2)-(4) represent the optimal delivery budget, including process budget and budget that depart from time windows [12]. $r$ and $i$ represent the number of delivery locations and the number of transportation vehicles.

Equation (5) represents the load range of the transportation vehicle. Equation (6) represents the number of receipts at the customer receiving location. It can be guaranteed that only one vehicle transports goods to the location. Equation (7) and equation (8) represent the assignment of tasks to 
transportation vehicles. Equation (9) represents the breadth of time window across customer locations. Equation (10) and equation (11) decides that each logistics transportation vehicle must be sent out from the centralized warehouse, and they will return to the centralized warehouse after completing the machinery transportation task.

Then, the characteristics of the logistics vehicle routing problem model with time windows are analyzed. This research will improve the genetic algorithm from four aspects.

First, the improvement of the chromosome coding method. Within the genetic algorithm, different coding methods correspond to specific calculation methods, which determines how to optimize the algorithm.

Therefore, the first condition for optimizing the genetic algorithm is to change the coding method of the chromosome. The current general method of using the genetic algorithm for conditional limit calculation is binary method and natural number chromosome coding [13]. The binary method is universal because its calculation process is relatively simple. However, facing a relatively large-scale logistics transportation routes, it is difficult to calculate, and the subsequent calculation process will produce an unsolved situation. Therefore, the binary method is not suitable for calculation of logistics vehicle routing planning.

The research uses digital coding to optimize the algorithm, and expresses the delivery location specified by the customer in the range of $(1,2, \ldots, n)$. This range can be viewed as a chromosome congression. Each gene in the congression corresponds to a customer, and a representative centralized warehouse (0) is added to the chromosome sequence to distinguish each different sub-route. For example, 025680 represents the transportation vehicle to the customer's location 2 , $5,6,8$ in turn for machinery transportation, with returning to the centralized warehouse.

Second, the adjustment of the convergence rate. Adjacent search method is used to determine the location of a customer and search for the next adjacent location until the searched location meets the required number of transportation vehicles.

A customer location is randomly selected as the initial location, and it is used as a base point to search for the adjacent customer location with the shortest distance as the subsequent location. Then, the customer location with the shortest distance from the above location is continued to search, until the searched customer location meets the maximum capacity of the transportation vehicle. In this way, different sub-routes are formed to complete the allocation of customer locations.

The new chromosomes and new population base points are established. It will make the calculation method quickly approach the best answer at the beginning, thereby improving the optimization and convergence rate of the algorithm [14].

Third, the survival of the fittest. The good genes are genetically evolved, and the bad genes are eliminated. The most widely used method for selecting good genes is the turntable selection method [15]. The turntable is divided into unequal regions, and the direction the pointer points is determined by the size of the region. Therefore, in the genetic algorithm, the adaptive capacity within the population varies with individual changes. It is calculated whether the individual will be selected according to the proportion of the individual's adaptive capacity in the total capacity range. It is assumed that the total number of individuals of the population at the beginning is $M$. The adaptive capacity of the individual $n$ is $f_{n}$. The adaptive capacity $f_{n}$ of all individual is calculated to obtain total adaptive capacity of the population.

$$
\sum_{n=1}^{M} f_{n}
$$

Then, the probability that an individual is selected for heredity is:

$$
P_{n}=\frac{f_{n}}{\sum_{n=1}^{M} f_{n}}
$$

Then, the overlapping probability of each chromosome is:

$$
v_{n}=\sum_{x=1}^{n} P_{n}
$$

The advantage of the turntable selection method is that the operation is simple, but its randomness is strong. Many good genes in the population may not be selected, thus, the process of getting the best answer can be difficult. Therefore, this research will preserve the excellent chromosomes on this basis. When using the turntable selection method for screening, the excellent chromosomes saved before the screening are used to replace the poor chromosomes after the screening. It enables the stable inheritance of good genes, improves the efficiency of the calculation method, and the ability to find the best answer.

Fourth, cross-exchange. To avoid the loss of good genes in the genetic process to reduce the calculation capacity of the genetic algorithm, this research uses the method of maximal preservation of good genes to cross-exchange certain genes to ensure stable inheritance of good genes [16]. First, two genes on two chromosomes that contain an equal number of " 0 " are randomly selected as interchangeable samples. That is, the two genes contain the same number of centralized warehouses. The result of the cross-exchange is that the overlapping part pairs of the two genes are automatically deleted, and the other genes are filled in the deleted parts to form a new gene segment. It is shown in Figure 3. 


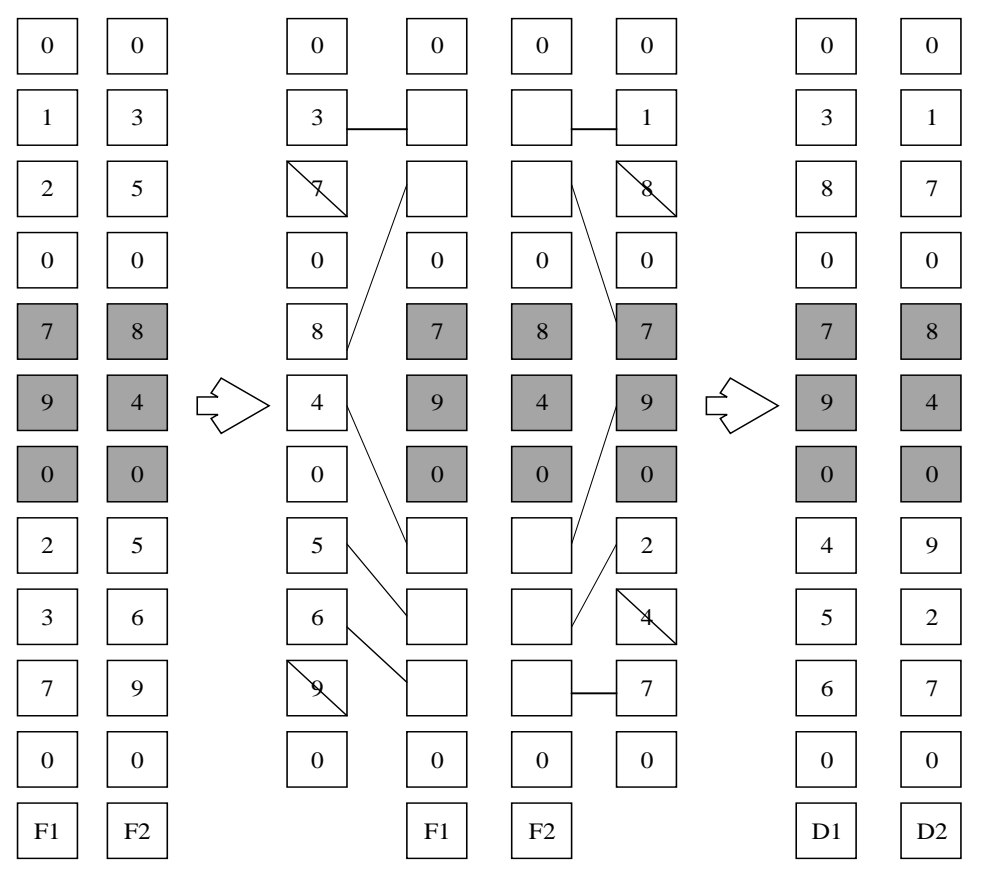

A

B

$\mathrm{C}$

Figure 3: The chromosome gene cross-exchange diagram (A: Gene segment selection; B: Overlapping genes deletion; C: Filial generation chromosome)

Fifth, gene change. When entering the final step of the calculation, the chance of chromosomes forming good individuals in the cross-exchange process is very small [17]. But the actual good number does not reach the expected value. Therefore, the method of gene change is used to produce more excellent individuals, finding the best number of expected values. This paper proposes a method for unregulated insertion of gene changes. If the gene change probability of a chromosome is $\mathrm{P}_{0}$, and the first gene of each chromosome is fixed, the judgment of whether the remaining genes are changed is based on the randomly selected floatingpoint number $\mathrm{E}_{\mathrm{i}}$ from the [0.1] set. If $\mathrm{E}_{\mathrm{i}}<\mathrm{P}_{0}$, the gene is removed from the chromosome and stored in the gene bank. If $\mathrm{E}_{\mathrm{i}}>\mathrm{P}_{0}$, the gene does not change.
After all the genes have been identified, a gene is randomly selected from the gene bank and inserted into any other place where the chromosome is not first. At the same time, it must be ensured that the lines transported by vehicles will not be overloaded. If the line load is exceeded, the gene needs to be adjusted to another location. The above steps are repeated continuously until all genes in the gene bank have been inserted, which means that the gene change is successful.

\subsection{The optimized genetic algorithm simulation}

First, the number of customer locations is 10 .

The location information is shown in the table below.

Table 1. Customer Information

\begin{tabular}{|c|c|c|c|c|}
\hline $\begin{array}{c}\text { Customer location } \\
\text { number }\end{array}$ & $\begin{array}{c}\text { Geographical } \\
\text { position }(\mathrm{km})\end{array}$ & $\begin{array}{c}\text { Machinery } \\
\text { requirements }(\mathrm{t})\end{array}$ & Time consuming (h) & Time window (h) \\
\hline $\mathrm{a}$ & $(4.9,12.8)$ & 0 & 0 & {$[0,24]$} \\
\hline $\mathrm{b}$ & $(2.8,4.9)$ & 1.4 & 1 & {$[2.8,9.8]$} \\
\hline $\mathrm{c}$ & $(13.9,11.4)$ & 0.5 & 1 & {$[3.1,7.5]$} \\
\hline $\mathrm{d}$ & $(16.5,11.8)$ & 0.4 & 1 & {$[4.1,10.2]$} \\
\hline $\mathrm{e}$ & $(10.1,7.9)$ & 1.3 & 1 & {$[3.9,7.8]$} \\
\hline $\mathrm{f}$ & $(6.9,8.9)$ & 0.9 & 1 & {$[4.0,9.1]$} \\
\hline $\mathrm{g}$ & $(4.9,9.1)$ & 0.7 & 1 & {$[6.1,11.5]$} \\
\hline $\mathrm{h}$ & $(0.8,10.5)$ & 1.2 & 1 & {$[0.5,10.8]$} \\
\hline $\mathrm{i}$ & $(6.1,16.5)$ & 1.1 & 1 & {$[2.5,5.9]$} \\
\hline $\mathrm{j}$ & $(7.1,18.5)$ & 0.5 & & \\
\hline
\end{tabular}


In the above table, the code 0 represents the centralized warehouse. The customer's specific condition is to use the lowest number of transportation vehicles for machinery transportation, while the cost incurred during transportation is the least. The maximum load of the transportation vehicle is $6 \mathrm{t}$. The transport distance cannot exceed $60 \mathrm{Km}$. The speed of transportation is kept at $25 \mathrm{Km} / \mathrm{h}$. If the transportation vehicle violates the time window, the penalty rule is: the vehicle is punished $2 \mathrm{Km} / \mathrm{h}$ for early arrival, and the vehicle is punished $4 \mathrm{Km} / \mathrm{h}$ for late arrival.

When loading and unloading a transportation vehicle, the working efficiency of the vehicle is reduced due to some external factors. Therefore, in order allocation, the first consideration should be the total number of transportation vehicles [18]. The calculation method is shown below.

$$
N=\left[\sum w_{a} / b E\right]+1
$$

Where: [] represents that all numbers in the equation that are calculated using integers, $b$ represents the parameter and $b \in[0,1]$. The more complicated the external factors encountered during the loading and unloading of the vehicle, the smaller the value of $b$ and the lower the working efficiency of the vehicle. The value of $b$ in this study is 0.6.

Then, the calculation steps of the optimized genetic algorithm are as follows (the Matlab software is used to calculate):
First, the numbers are marked to represent the number of chromosomes of the transportation vehicle route.

Second, the parameter value is specified. Q represents the range of population. $W$ represents the maximum of the filial generation of population evolution. $\mathrm{P}_{\mathrm{i}}$ represents the mixed probability. $\mathrm{P}_{\mathrm{k}}$ represents the probability of gene mutation. $H$ represents the number of the filial generation currently inherited. $L$ represents the genetic gap.

The specified location of the customer is $\mathrm{V}(\mathrm{o}, \mathrm{p})$.

Third, the new population is established.

Fourth, the given fitness calculation method is used to solve the size of the fitness.

Fifth, the number of the filial generation inherited is set to 0 .

Sixth, the specific selection method is used to screen individuals with excellent filial generations for next-generation genetic.

Seventh, the specific cross-mutation method is used for the exchange and change of genes.

Eighth, the new population generates.

Ninth, If the conditions for calculation stop are met, it is stopped immediately. If not, it needs to go back to step 4 to continue the calculation.

The above steps are used for calculation and generation-by-generation screening to finally obtain the optimal answer of the objective function.

That is, the optimal transportation route of the transportation vehicle. The transportation vehicle route with the time window limitation is solved.

The calculation steps of the above algorithm are shown in the following figure.

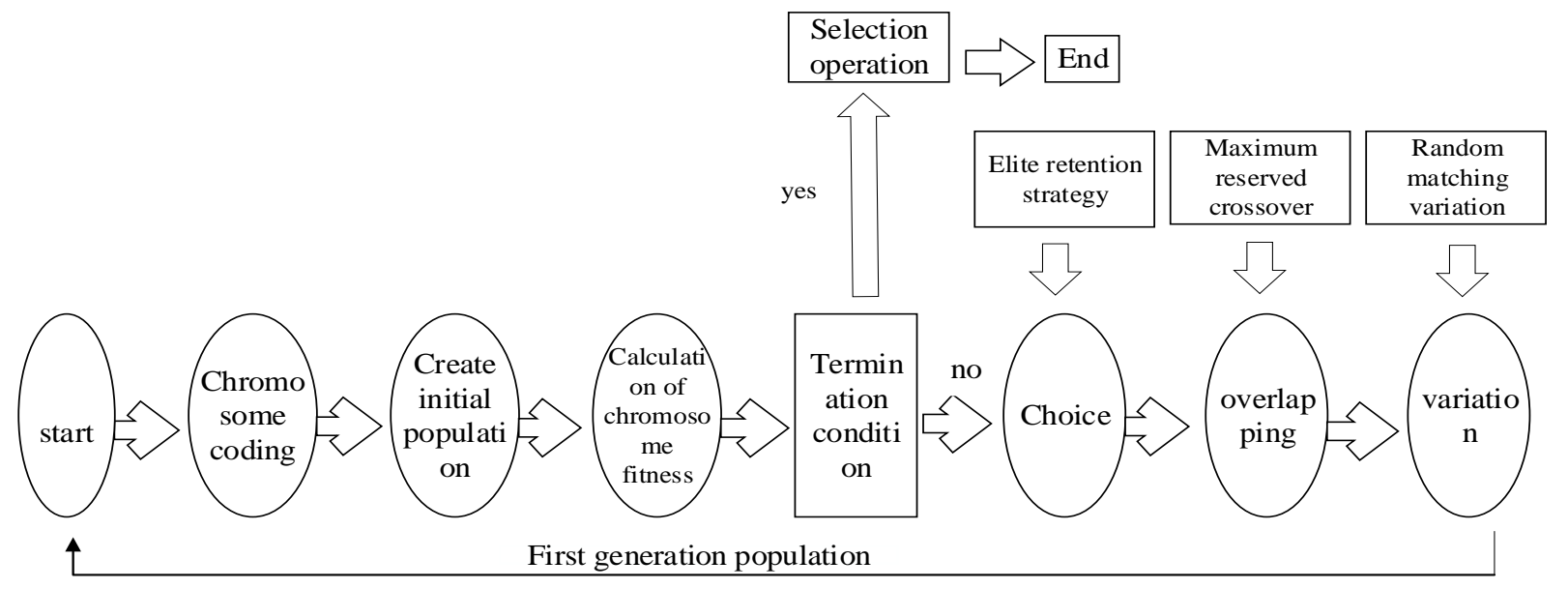

Figure 4: The calculation steps of the genetic algorithm

\section{Results}

\subsection{The comparison of transportation routes between optimized genetic algorithm and general genetic algorithm}

Comparing the 8 effective tests of the optimized genetic algorithm and the general genetic algorithm in Matlab, the lowest cost obtained is shown in the following table.

From the table above, the average distance cost calculated using the optimized genetic algorithm is $143.3 \mathrm{~km}$. The best route is sending 4 transportation vehicles for machinery transportation, and the total distance cost is $119 \mathrm{~km}$. 
Table 2. Results of 8 tests of optimized genetic algorithm and general genetic algorithm

\begin{tabular}{|c|c|c|c|c|c|c|c|c|}
\hline & 1 & 2 & 3 & 4 & 5 & 6 & 7 & 8 \\
\hline $\begin{array}{c}\text { optimized } \\
\text { genetic } \\
\text { algorithm }\end{array}$ & 149.3 & 146.2 & 141.5 & 135.8 & 140.8 & 136.7 & 147.4 & 148.9 \\
\hline $\begin{array}{c}\text { general } \\
\text { genetic } \\
\text { algorithm }\end{array}$ & 152.1 & 161.5 & 156.2 & 167.1 & 145.3 & 164.4 & 174.2 & 162.6 \\
\hline
\end{tabular}

The best route is sending 4 transportation vehicles for machinery transportation, and the total distance cost is $119 \mathrm{~km}$.

Then, 4 transportation routes are:

Route 1: 0-7-2-19-6-0

Route 2: 0-4-12-18-16-3-0

Route 3: 0-10-9-2-15-1-0

Route 4: 0-7-8-17-14-0

The best route of transportation vehicles is shown below.

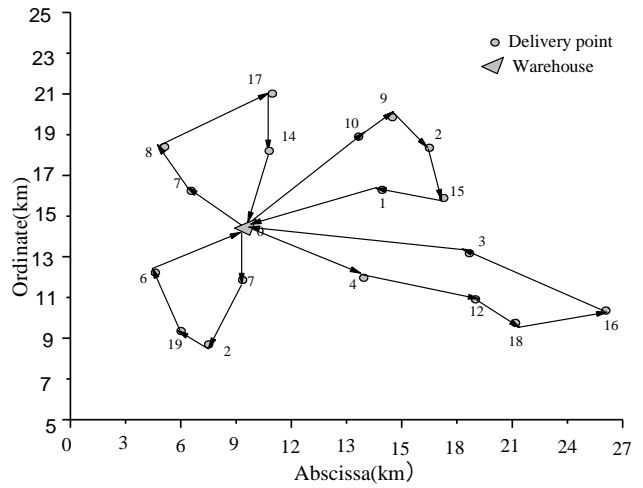

Figure 5: The best transportation route of the optimized genetic algorithm

\subsection{The comparison of the calculation process of optimized genetic algorithm and general genetic algorithm}

In the calculation process of the optimized genetic algorithm, with the increase in the number of genetic generations, the number of good genes is reduced compared with the general genetic algorithm.

The optimization comparison of the genetic algorithm process is shown below.

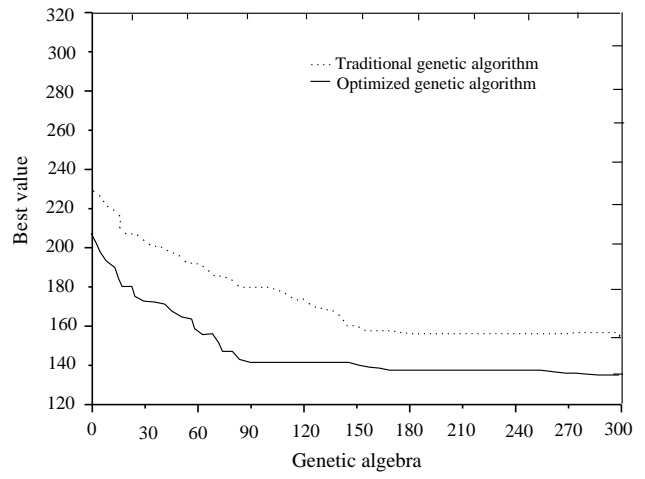

Figure 6: The optimization comparison of the genetic algorithm process

\subsection{The comparison of the calculation efficiency of the optimized genetic algorithm and the general genetic algorithm}

The results of 8 tests are analyzed. Compared with the general genetic algorithm, the optimized genetic algorithm reduces the average transportation cost and the maximum transportation cost, improving the search success rate of the best route.

The efficiency comparison results of the two are shown in the following figure.

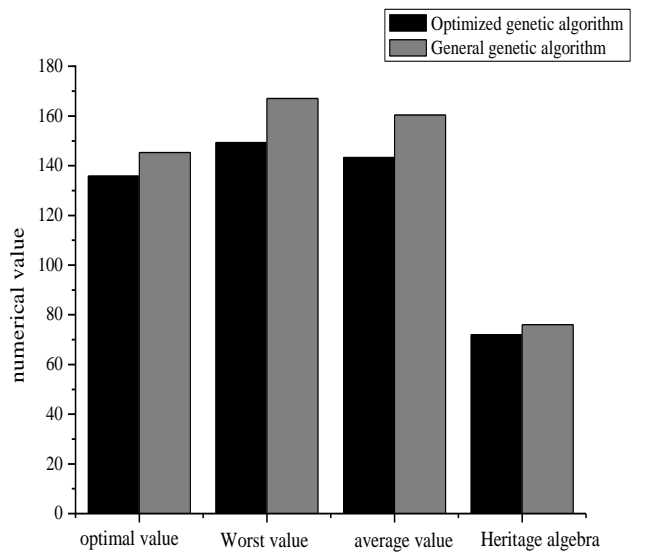

Figure. 7: The efficiency comparison of genetic algorithm

From the figure, when planning the route of vehicles in the actual machinery production logistics, the general genetic algorithm will cause the loss of good genes due to the shortcomings of the gene cross-exchange and change methods. It will lead to low calculation efficiency.

The optimized genetic algorithm can effectively solve the problem of good gene loss, and the calculation efficiency is significantly improved.

The best vehicle transportation route is planned to maximize cost savings while meeting customer requirements.

\section{Discussion}

With the improvement of comprehensive national strength and economic strength, China's logistics industry has also developed rapidly.

For logistics itself, logistics distribution capability is the key to the development of this field. Logistics distribution is also the route planning of logistics 
vehicles, and its purpose is to use the lowest delivery cost to complete the distribution task [19].

Therefore, it is necessary to develop a logistics transportation route that can intelligently complete fast and accurate logistics transportation according to customer needs, thereby creating considerable benefits for logistics enterprises.

However, in the actual logistics transportation process, the customer will have a transportation time window [20] limitation.

Or because of external factors such as complicated weather and roads, the logistics transportation scheme needs to be scientifically optimized.

This paper improves the algorithm for the logistics transportation route in the machinery production logistics system. The genetic algorithm in data mining is optimized to make the transportation route have good applicability and extensiveness.

At the same time, it solves the problem that genetic algorithms are susceptible to regional optimization and slow convergence under complex paths. However, the machinery logistics transportation route with time window limitation studied in this paper is only a relatively simple allocation of transportation vehicles. It cannot really solve the problem of the complex scenario transportation routes in the actual machinery logistics system. Therefore, it is necessary to conduct research on logistics vehicle allocation and route planning with more complicated customer conditions for more transportation vehicles and warehouses. At the same time, on the problem of algorithm optimization for data mining, this study only optimizes the genetic algorithm with the single optimization object.

Other algorithms should also be optimized to find the best solution for transportation vehicle route planning in the machinery production logistics system.

\section{Conclusions}

China's research on the route of transportation vehicles in the logistics system started relatively late. However, with the rapid development of the Internet of Things, the level of the logistics industry is also increasing rapidly. Many domestic researchers have proposed methods that can efficiently solve the problem of transportation vehicle route planning. It can solve the problem of algorithm optimization in the course of route calculation, and greatly improve the efficiency of vehicle transportation.

This paper studies the transportation vehicle route planning problem in the machinery production logistics system. The genetic algorithm in data mining is used to change the initial population establishment method and optimize the way of gene cross-exchange and change.
And the transportation vehicle route is calculated, obtaining the result of applicability and efficiency. Then, the simulation experiment of vehicle transportation is carried out in the machinery logistics system.

Comparing the general genetic algorithm with the optimized genetic algorithm, the results show that the optimized genetic algorithm has higher efficiency and adaptability.

\section{References}

[1] Zhang W. J., An Y. (2015) Abnormal ionization in sonoluminescenceProject supported by the Research Fund for the Doctoral Program of Higher Education of China (Grant No. 20120002110031) and the National Natural Science Foundation of China (Grant No. 11334005). Chinese Physics B, 24(4), 8-10.

[2] Daniel A. Russell. (2016) Things I learned while teaching a graduate level course on the acoustics of musical instruments. Journal of the Acoustical Society of America, 139(4), 2096-2096.

[3] Chandrika K. (2016) Data mining and statistical inference in selective laser melting. International Journal of Advanced Manufacturing Technology, 86(5-8), 1659-1677.

[4] Cheng A., Qu T., Wang Z. Z. (2015) Optimal Configuration of Transportation Resources Based on Queuing Theory in a Production Logistics System. Journal of Guangdong University of Technology, (12), 38-43.

[5] Lv K., Liu Y. (2016) Modeling and Simulation of Flowers Production Logistics Based on Petri Net, 16, 67-69.

[6] Zhang Y. F., Member, IEEE. (2018) A Framework for Smart Production-Logistics Systems based on CPS and Industrial IoT. IEEE Transactions on Industrial Informatics, 14(9), 4019-4032.

[7] Chen K. H., Nguyen Q. C., Jiang S, et al. (2016) Application of route flexibility in data-starved vehicle routing problem with time windows, 2 , 102-108.

[8] Yang Y. W., Lu E. H. C. (2017) Pool-based Recursive Construction Approach for Logistics Route Planning with Time Window, 2017 6th IEEE International Conference on Advanced Logistics and Transport (ICALT). IEEE, 18, 221225.

[9] Hu C., Lu J., Liu X. (2018) Robust vehicle routing problem with hard time windows under demand and travel time uncertainty. Computers \& Operations Research, 94, 168-172.

[10] Masatoshi K., Noh N. J., Taku M. S. (2017) Current and future carbon budget at Takayama site, Japan, evaluated by a regional climate model and a process-based terrestrial ecosystem model. International Journal of Biometeorology, 61(6), 989-1001. 
[11] Huang M. H., Cheng Z. H. (2016) A longitudinal comparison of customer satisfaction and customer-company identification in a service context. Journal of Service Management, 27(5), 730-750.

[12] Badu A. B., Fakorede M. A. B., Talabi AO. (2015) Gene action and heterotic groups of early white quality protein maize inbreds under multiple stress environments. Crop Science, 56, 183199.

[13] Fernández-Miñán A., Bessa J., Tena J. J. (2016) Assay for transposase-accessible chromatin and circularized chromosome conformation capture, two methods to explore the regulatory landscapes of genes in zebrafish. Methods in Cell Biology, 135, 413.

[14] Ji L. B., Chen F. (2017) Process optimization of rolling for zincked sheet technology using response surface methodology and genetic algorithm. International Journal of Modern Physics B, 31(16-19), 1744017.

[15] Jing L., Chi Y. X., Chen Z. (2016) A Dynamic Multiagent Genetic Algorithm for Gene Regulatory Network Reconstruction Based on
Fuzzy Cognitive Maps. IEEE Transactions on Fuzzy Systems, 24(2), 419-431.

[16] Byung S. K., Cheol M. J. (2015) Scheduling Trucks in Multi-Door Cross Docking Systems: An Adaptive Genetic Algorithm with a Dispatching Rule. Asia Pacific Journal of Operational Research, 32(3), 1550016.

[17] Hudda N., Fruin S. A. (2017) Carbon dioxide accumulation inside vehicles: The effect of ventilation and driving conditions. Science of the Total Environment, 610-611, 1448-1456.

[18] Tao Y., Chew E. P., Loo H. L. (2016) A column generation approach for the route planning problem in fourth party logistics. Journal of the Operational Research Society, 68(2), 165-181.

[19] Chen X. L., Mark L., Gestring M. R, Rosengart. (2018) Logistics of air medical transport: When and where does helicopter transport reduce prehospital time for trauma, Journal of Trauma \& Acute Care Surgery, 85(1), 1.

[20] Faissal A. M. S., Besher A. A., Mohammad K. (2018) Impact of Logistics with the Cold Ischemia Time of Utilized Deceased Organs in KSA. Transplantation, 102, 770. 